\title{
The Stable Image System and Method of Quick Reflective Mirror based on Related Detection of Pictures
}

\author{
Huihua Jiao \\ Information technology department \\ QiongTai Teachers College \\ Haikou,China \\ xueerjhh@qq.com
}

\author{
Wei Zhou \\ Shanghai institute of physics \\ Chinese academy of sciences \\ Shanghai,China \\ zw-1221@163.com
}

\begin{abstract}
The stable image system and method of quick reflective mirro based on related detection of pictures means the stable tracing system to the trembling light beam in action or trembling environment through diversive light beam of the quick reflective mirro. The system firstly detect the offset of the unstable light beam on real time through image related methods and send the feedback of the off set of light beam to the controller of the quick reflective mirro, and control the diversion of the quick reflective mirro on real time, thus realize the stability of the light beam. The sysytem can realize the stability of single frame image, avoid the obscurity of pictures caused by light beam tremble during the integral time of the high resolution camera, and improve the imaging quality of the image forming system.
\end{abstract} FPGA

Keywords-Imagestabilizationzsystem; Imagedetection; CMOS;

\section{INTRODUCTION}

This article relates to optical imaging system, image stabilization, the camera in the imaging process, due to various external or self-interference, resulting in the exposure time, the image relative motion in the focal plane, the image quality is often a certain degree of degradation In order to obtain high-resolution images, image stabilization measures must be taken. Image stabilization technology makes the overall performance of the application of the imaging system has been improved, imaging resolution is greatly improved. Real-time image stabilization technology plays an important role in the military satellite camera battlefield reconnaissance, target surveillance and earlywarning monitoring[1].

Airborne, shipboard and space imaging systems for imaging target, due to the motion platform, flutter and attitude change in the exposure time of the camera image and the photosensitive element relative motion of the illuminated object, and thus bring the imaging blur smearing, making the image quality is often a certain degree of degradation of contrast and resolution reduction, for high-resolution cameras, the situation is even more so. This degradation alone can improve the resolution of the imaging device or simply post-image processing can not meet the requirements to obtain high-resolution image quality, and therefore, how to reduce or even eliminate the influence of external interference on the image quality has become a key technology where image stabilization .

Image stabilization technology currently used are: optical image stabilization technology, micro-mechanical image stabilization technology, electronic image stabilization technology and integrated image stabilization technology. Optical image stabilization is to set some of the optical elements in the optical path to compensate for unstable images[2]. A typical method is to use optical image stabilization wedge to control the direction of the line of sight, by moving or rotating optical wedge, change the angle and direction of light rays, for image motion compensation, so as to achieve the purpose of image stabilization. The main drawback of optical image stabilization technology is only applicable to a small vibration environmental conditions, and the compensation structure and manufacturing process is too complicated[3]. Also, if you only rely on the optical element prisms, mirrors and other optical wedge or passive compensation, stable capability are more restricted. Electronic image stabilization technology is a method of digital image processing of image sequences directly determine the offset and compensate technology, electronic image stabilization method is to remove the scrambled video image sequences inter unintentional camera shake caused dueMoving, it only allows video sequence during playback to produce a stabilizing effect in the visual playback, but not for single image stabilization during the exposure time, which is not really improve its image resolution. Micromechanical image stabilization technology is directly controlled by the micromechanical deflection mirror means for rotating or moving the focal plane imaging, image motion compensation, complete stabilization tasks. Micromechanical image stabilization system is simple, compact, but need to get the position and displacement of the focal plane is controlled by a certain method. Integrated image stabilization technology is the application of a variety of image stabilization technology together to achieve stabilization to obtain more like a qualitative technique[4]. 
Image stabilization system described herein belong micromechanical image stabilization systems, micromechanical image stabilization mode is the overall improvement of the traditional way of image stabilization ray machines, high-speed, high-precision image motion detection capabilities and compensation implementation capacity, and use of the technology System components also has a small size, low power consumption, the impact on small and remote sensing optical system little effect on the image quality and many other advantages, it has become one of the micro-mechanical image stabilization technology to compensate high-resolution remote sensing is preferred.

\section{IMAGE STABILIZATION SYSTEM}

architecture of the system architecture block diagram shown in Figure 1.

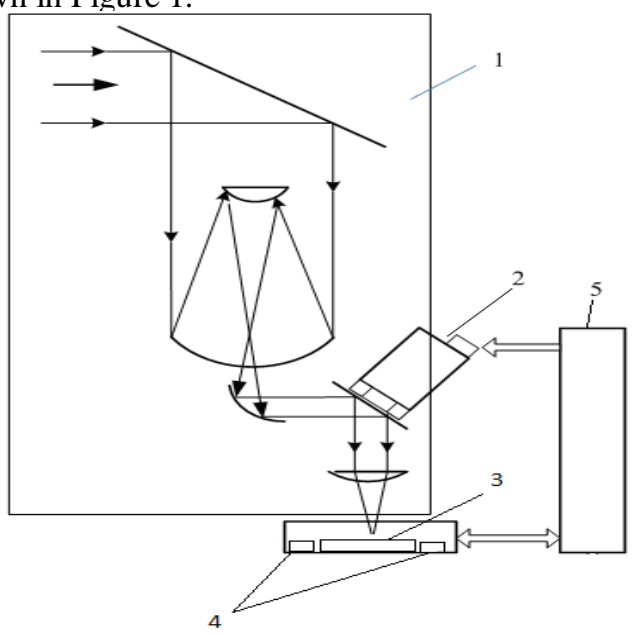

Figure 1. Fast mirror image correlation detection of a 1-image stabilization system imaging optical system structure diagram

The main processing unit of the entire system by the imaging optical system,1. rapid mirror 2, Omo focal plane array detectors 3 , two miniature high-speed CMOS sensors 4 and 5 FPGA image processing unit related components[5]. The key to this system is that the mini-high-speed CMOS detector how to get real-time imaging system optical axis offset, because the optical axis of the imaging system when the cause jitter, on the focal plane of the image will be slight bias occurred due to external disturbance or own motion move, this time to install high-speed CMOS detectors in the focal plane, arranged in parallel with the main imaging detector, in order to improve the accuracy of image shift amount detected, this uses two miniature CMOS detector mounted side by side in the front focal plane of the Omo both sides of the detector focal plane array detectors and Omo distance of not more than 2mm, as shown in FIG.

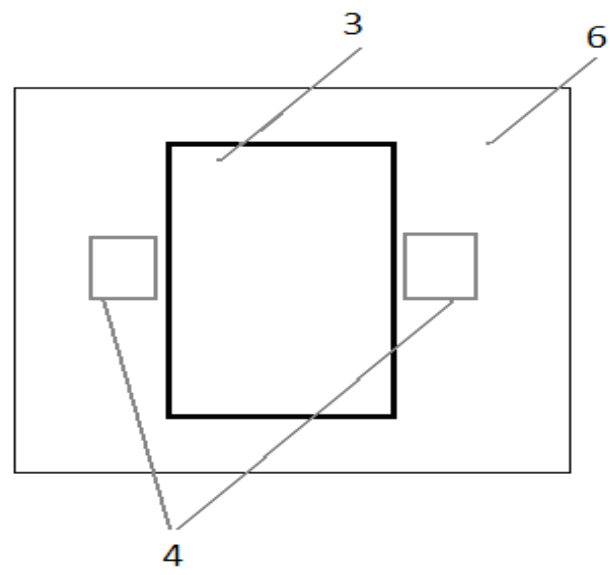

Figure 2. The focal plane detector imaging system installation diagram

6- focal plane imaging system of FIG. 2

miniature CMOS image detector for high-speed sampling, and real-time image correlation method for obtaining an image in the focal plane of the offset[6]. The optical signal passes through the imaging optical system has been fast mirror will signal reflections gathered in large-array focal plane array detectors and two auxiliary miniature high-speed CMOS sensor above, two auxiliary miniature high-speed CMOS image sensor for high-speed sampling campaign obtained by the image processing unit in real-time FPGA image correlation offsebecause the optical axis of the imaging system when the cause jitter, on the focal plane of the image will be slight bias occurred due to external disturbance or own motion move, this time to install highspeed CMOS detectors in the focal plane, arranged in parallel with the main imaging detector, in order to improve the accuracy of image shift amount detected, this uses two miniature CMOS detector mounted side by side in the front focal plane of the Omo both sides of the detector focal plane array detectors and Omo distance of not more than $2 \mathrm{~mm}$, as shown in FIG. Figure 2. The focal plane detector imaging system installation diagram6- focal plane imaging system of FIG. 2 miniature CMOS image detector for high-speed sampling, and real-time image correlation method for obtaining an image in the focal plane of the offset[7]. The optical signal passes through the imaging optical system has been fast mirror will signal reflections gathered in largearray focal plane array detectors and two auxiliary miniature high-speed CMOS sensor above, two auxiliary miniature high-speed CMOS image sensor for high-speed sampling campaign obtained by the image processing unit in real-time FPGA image correlation offset, offset rapid feedback to mirror controller to control the rapid deflection mirror so that the image stabilization in the focal plane. Fast mirror piezoelectric ceramic fast swing mirror, piezoelectric ceramics having a frequency response, high resolution, accurate displacement characteristics. Finally, the focal plane array detectors Omo stable image points, so that the focal plane array detector Omo high-resolution images can be obtained[8]. 
Image correlation algorithm than larger, but the high accuracy of the results, the error is less than 0.3 pixels. Large amount of calculation for image-related problems, the system is designed for fast image correlation-based FPGA module, FPGA parallel processing, flexible programming features[9]. The system FPGA image processing module associated fast Fourier transform method to achieve real-time two images related operations, its internal function module shown in Figure 3, its related processing flow is as follows:

1)By high-speed micro-CMOS image detector output is fed to the FPGA image input interface, first through FPGA image preprocessing unit, related algorithms for image preprocessing operations, namely undercurrent correction, gain correction.

2)import images from pretreatment, respectively, the reference frame image and the current frame image is performed in parallel FFT Fourier transform, where the use-2 fast Fourier transform algorithm to improve processing speed.

3)After the reference frame image and the current frame image FFT transform, and then the reference frame image taken conjugate FFT, FFT frame image with the current direct multiplication, the result was then multiplied by the inverse Fourier transform IFFT, This completes the two images related operations.

4.Finally, surface fitting, whichever calculation results in the maximum correlation location, locationCoordinate is the position offset between two frames by output interface to the FPGA fast mirror drive control input. Complete offset of feedback control. The system is a high-speed FPGA image correlation methods to achieve motion detecting image offset, and then using the rotation control micromechanical device image stabilization fast mirror in the focal plane, the system has fast response, small size, compact structure, etc.

\section{THE IMPLEMENTING TECHNOLOGY RELATED TO THE SYSTEM}

In order to realize the stabilization image system, we use the implementation technology as follows:

1)The fast mirror image system based on image correlation detection system, mainly refers to the focal plane imaging system on both sides of the two auxiliary micro high-speed CMOS detector to sample in real time, the sampling of two adjacent frames image through the image correlator based on FPGA calculating image motion vector, will give mirror motion vector feedback to the fast controller, and to control the reflector deflection beam, then to realize a stable imaging[10].

2)We use two auxiliary micro high-speed CMOS detector to sample focal plane migration image, the probe' $\mathrm{s}$ pixel size is within the $64 \times 64$, frame rate is higher than 120 frames per second, the two micro detector are installed in the main image on both sides of the detector, the distance between detector and imaging is less than $2 \mathrm{~mm}$, and confocal with the main imaging detector.

3)Using FPGA to realize two frames 64 x64 pixel images processing, using fast Fourier transform algorithm to calculate the offset of the two frames fast.
4)The fast mirror to control the beam deflection which based on piezoelectric ceramic, fast reflector controller' $s$ input and image correlator image' s offset of the correlator output cascade, using the PID control algorithm to realize the fast reflector closed-loop control.

\section{IMPLEMENTATION SCHEME}

According to this article, the fast mirror image system based on image correlation detection system, the structure diagram is shown in figure 1.The imaging optical system platform 1, the fast reflector 2, large-area FPA 3 , two miniature high-speed CMOS detector 4 and the FPGA image correlation processing unit 5.

Imaging optical system 1 use common optical telescope, diameter is $150 \mathrm{~mm}$, the focal length is $1800 \mathrm{~mm}, \mathrm{~F}$ number is 12 , resolution $<5$, "system instantaneous field of view is 8 urad, total 0.95 o field;

Fast reflector 2 adopts four piezoelectric deflection pivot XY platform, the closed-loop Angle can reach $+2 \mathrm{mrad}$, resolution of 0.05 urad, mirror diameter is $50 \mathrm{~mm}$, closed loop linearity $0.2 \%$, resonant frequency of $3.3 \mathrm{KHz}$, the distance between the lens and the focal plane $\mathrm{H}$ is $100 \mathrm{~mm}$;

Large-area FPA 3 adopts monochromatic plane array CCD devices, with area array size is $1 \mathrm{k} * 1 \mathrm{k}$, the response wavelength is $400 \sim 1000 \mathrm{~nm}$, pixel size is $14 \mathrm{um} * 14 \mathrm{um}$, frame frequency is $5 \mathrm{fps}$, the integral time can be adjusted;

Miniature high-speed CMOS detector 4 adopts high frame frequency small plane array CMOS devices, the array size is $64 * 64$, the visible light band, image motion size is $8 \mathrm{um}^{*} 8 \mathrm{um}$, frame frequency $150 \mathrm{fps}$, integration time is less than $1 \mathrm{~ms}$;

The FPGA image correlation processing unit 5 is shown in figure 3.

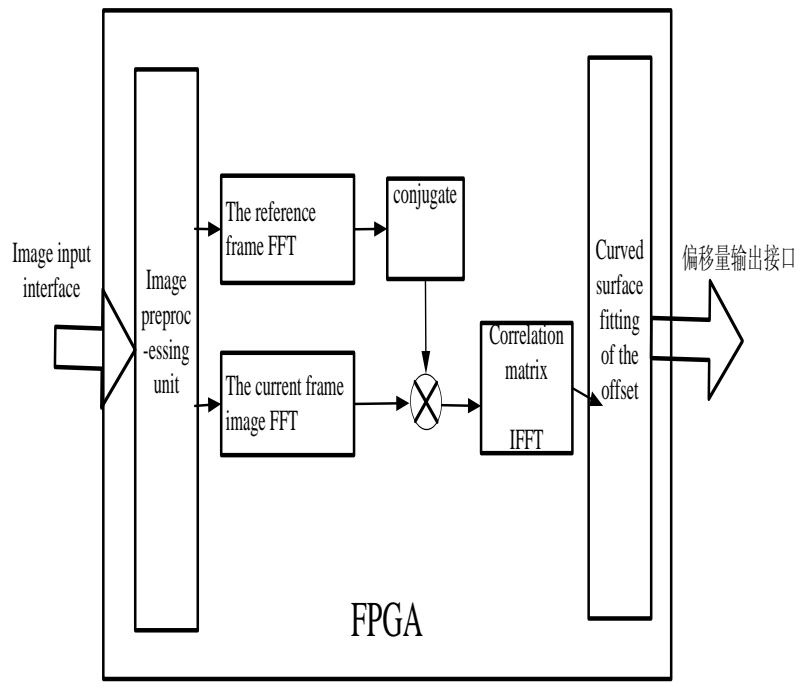

Figure 3. The FPGA image correlation processing unit function block diagram

It was consisted of image preprocessing module, image FFT transform module, the matrix IFFT inverse transformation module and curved surface fitting modules. The FPGA logic unit number is 12060 les, built-in 2 PLL and 234 kbits RAM, external clock is 40 MHZ. 


\section{CONCLUSION}

Described in this article, stabilization image system belongs to the Micro mechanical stabilization image system. The Micro mechanical stabilization image's way is through the total improvement on the traditional stabilize the image light machine, the new one has high speed, high precision of image motion detection ability and executed compensation ability, and using this technology of system has small volume, low power consumption, small influence on the optical remote sensor system and small effects on image quality advantages, so the micro mechanical compensation mode becomes one of the first selection of high resolution remote sensing apparatus stabilization image technology.

\section{REFERENCES}

[1] XU Zhi-hai,CHEN Yue-ting,WANG Qi,LIU Zhao-jun,LI Qi., Array space camera steady like compensation research $[\mathrm{J}]$. Infrared and Laser Engineering,. 2007 (1) ,pp.593-596

[2] GE Ren-wei,WU Qing-wen,WANG Yun1,CHEN Li-heng, Joint transform correlator as shift measurement test system design [J]. Chinese Journal of Optics and Applied Optics. 2009(3),pp. 218-224
[3] MA Yan-nan,XIE Hong-liang, The CCD image processing system based on DSP + FPGA is studied[J]. Telecommunications for Electric Power System,2007 (11).pp.234-236

[4] SHENG An;DONG Nan;ZHANG Bo;XU Zheng-yi;WEI Jian-ming. Application of fuzzy clustering to stabilize the image for electronic motion estimation algorithms[J], Application Research of Computers,2015(2),pp.598-602

[5] ZHeng Xiao-feng. Based on the motion detecting light compensation technology to stabilize the image of the machine[D]. Doctor and Master Thesis, 2007

[6] Li Haoyang Liu Zhaojun Xu Pengmei. Introduction to technology to stabilize the image space optical remote sensor[J]. Spacecraft Recovery \& Remote Sensing ,2010(2),pp.52-56

[7] ZHAO Dao-xin. DMMS and indicating instrument computer verification and management system [J]. ELECTRICAL MEASUREMENT \& INSTRUMENTATION,. 1994(5)

[8] LIU Zhi chun, WANG Jing ru,Xin Huang. Based on the FPGA design of image enhancement $[\mathrm{J}]$. Opto-electronic Engineering,2001 (3),pp.48-51

[9] FENG Hai-qi, The hardware implementation of real-time image enhancement processing $[\mathrm{J}]$. Radio Communications Technology, 1998(3),pp.108-112

[10] LI Yong-cai. Real-time infrared image enhancement processing technology research [D]. 2006 